Supplementary Information

\title{
Cloud Water Chemistry Associated with Urban Aerosols: Rapid Hydroxyl Radical Formation, Soluble Metals, Fe(II) and Fe(III) and Quinones
}

Xiaobi M. Kuang, ${ }^{\S, 1}$ David H. Gonzalez,, J. Adlin Scott,, Kennedy Vu, ${ }^{2}$ Alam Hasson, ${ }^{2}$ Tiffany Charbouillot, ${ }^{\S}$ Lelia Hawkins and Suzanne E. Paulson ${ }^{\S, *}$

sDepartment of Atmospheric and Oceanic Sciences, University of California at Los Angeles, Los Angeles, CA, 90095-1565 USA

${ }^{1}$ Now at: South Coast Air Quality Management District, Diamond Bar, CA, 91765 USA

${ }^{2}$ Department of Chemistry, California State University Fresno, Fresno, CA, 93740 USA

${ }^{3}$ Department of Chemistry, Harvey Mudd College, Claremont, California 91711, United States

\section{Mass Concentration}

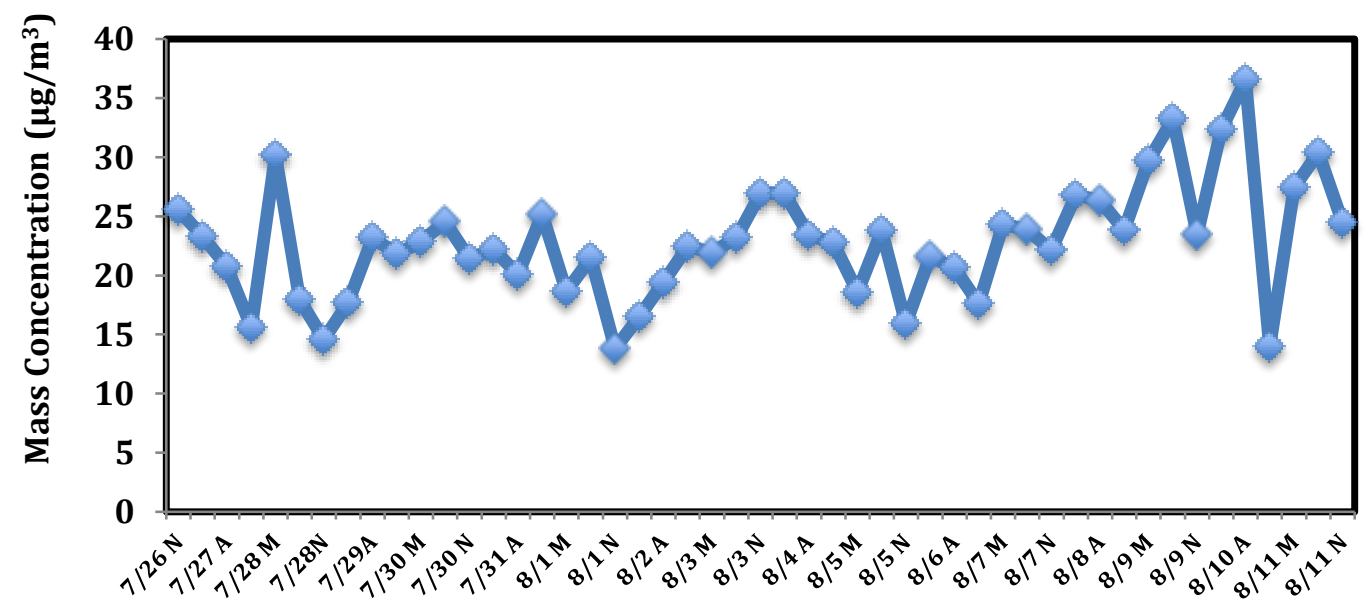

Figure S1. Mass concentration over the collection period in Claremont, CA, 2012. 


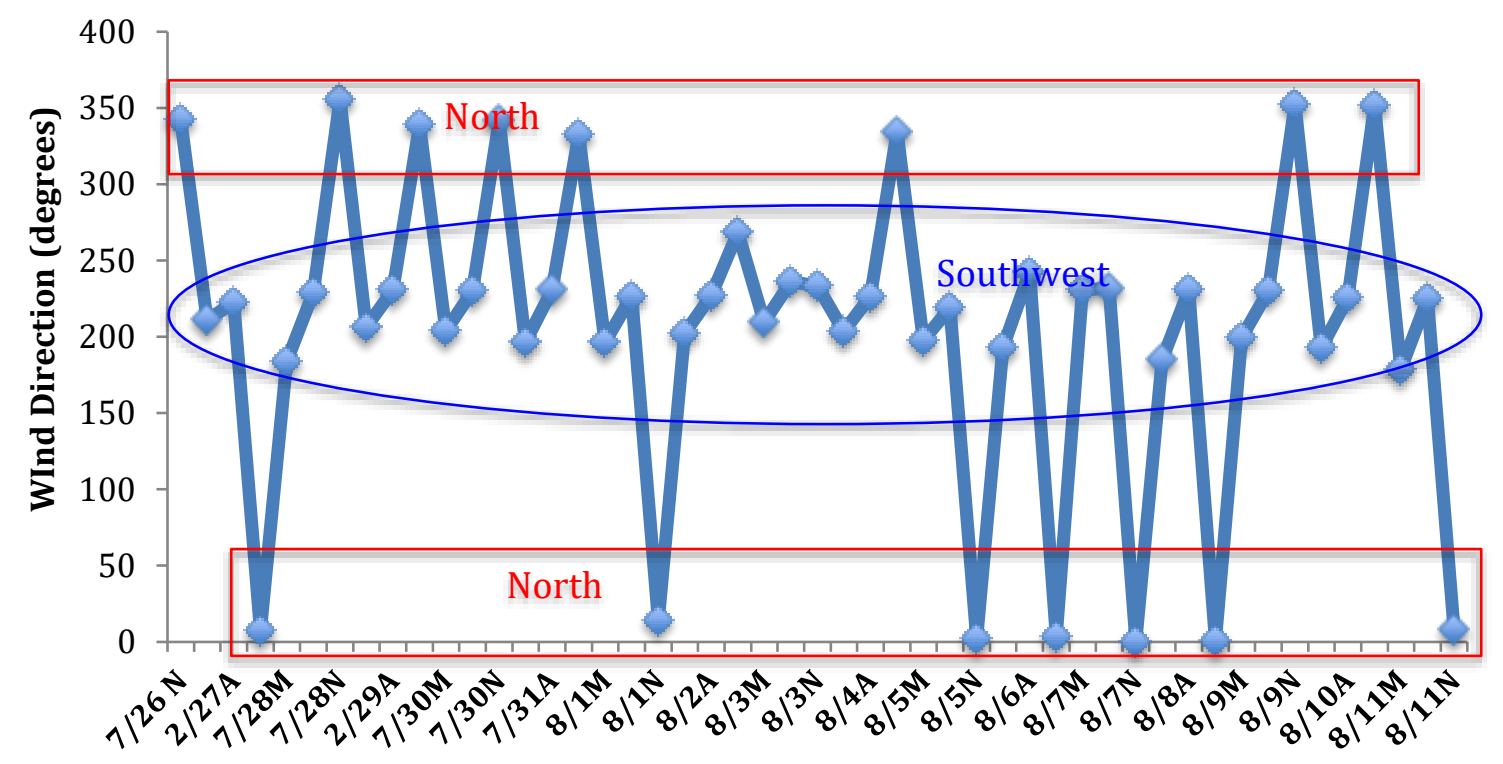

Figure S2. Wind direction over the collection period in Claremont.

Table S1. Mean limits of detection for quinones.

\begin{tabular}{ll}
\hline Quinone & LOD $\left(\mathrm{ng} / \mathrm{m}^{3}\right)$ \\
\hline Anthraquinone: & $1.6 \times 10^{-2}$ \\
\hline Benz-7,12-anthraquinone & $7.0 \times 10^{-2}$ \\
\hline Naphthacenequinone & $6.6 \times 10^{-2}$ \\
\hline Chrysenequinone & $1.6 \times 10^{-1}$ \\
\hline 1,2-Naphthoquinone & $7.5 \times 10^{-2}$ \\
\hline 1,4-Naphthoquinone & $5.3 \times 10^{-3}$ \\
\hline Phenanthraquinone & $2.0 \times 10^{-3}$
\end{tabular}



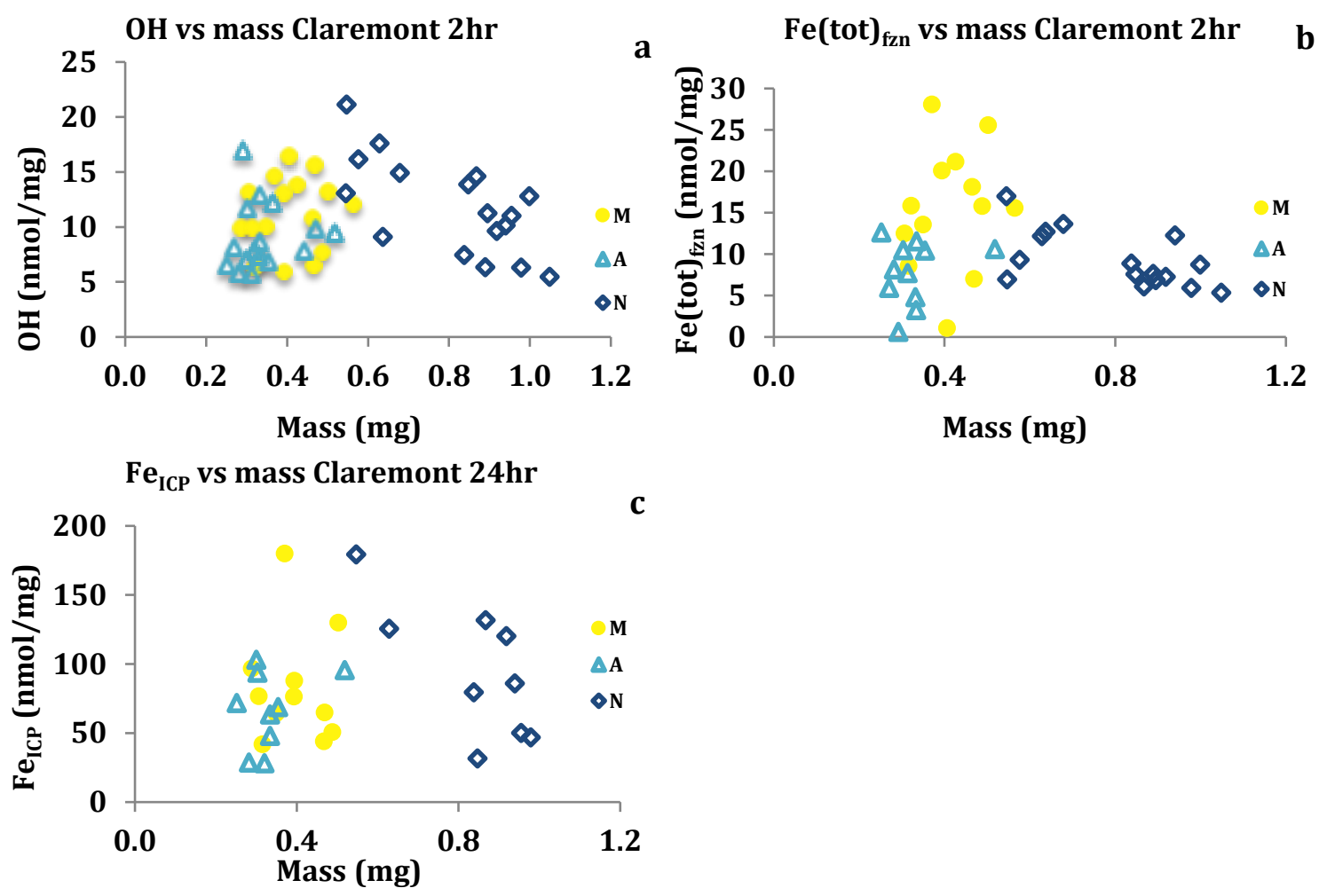

Figure S3. Mass-normalized $\mathrm{OH}(\mathrm{a}), \mathrm{Fe}(\mathrm{tot})_{\mathrm{fzn}}(\mathrm{b})$ and Feicp (c) vs mass to check for saturation effects. Circle (M), triangle (A) and Diamond (N) represent morning, afternoon and nighttime samples, respectively. 
Table S2. Correlations between mass and non-mass corrected $\mathrm{OH}$ initial concentration, and iron speciation, Claremont. $\mathrm{m}$ is slope of the regression line. Orange, light blue and dark blue values represent morning, afternoon and nighttime correlations, respectively; and black values represent the entire data set. Only significant correlations $(\mathrm{p}<0.05)$ are shown.

\begin{tabular}{|c|c|c|c|c|c|c|c|c|c|c|c|c|c|c|}
\hline & & \multicolumn{3}{|c|}{ mass } & \multicolumn{3}{|c|}{ Ini. OH } & \multicolumn{3}{|c|}{$\mathrm{Fe}(\mathrm{III})$} & \multicolumn{4}{|c|}{$\mathrm{Fe}$ (tot) } \\
\hline $\begin{array}{l}\text { Ini. OH } \\
(\mu \mathrm{M})\end{array}$ & $\begin{array}{l}\mathrm{r}^{2} \\
\mathrm{p} \\
\mathrm{m}\end{array}$ & $\begin{array}{l}0.61 \\
0 \\
1.12\end{array}$ & $\begin{array}{l}0.26 \\
0.01 \\
0.70 \\
\end{array}$ & $\begin{array}{l}0.46 \\
0 \\
1.03\end{array}$ & & & & $\begin{array}{l}0.41 \\
0 \\
2.61\end{array}$ & & & $\begin{array}{l}0.14 \\
0 \\
0.86\end{array}$ & & & \\
\hline $\begin{array}{l}\mathrm{Fe}(\mathrm{II})_{\mathrm{fzn}} \\
(\mu \mathrm{M}) \\
2 \mathrm{~h} \\
\end{array}$ & $\begin{array}{l}\mathrm{r}^{2} \\
\mathrm{p} \\
\mathrm{m}\end{array}$ & $\begin{array}{l}0.02 \\
0.5 \\
0.02 \\
\end{array}$ & $\begin{array}{l}0.23 \\
0.06 \\
0.93 \\
\end{array}$ & & & & & & $\begin{array}{l}0.27 \\
0.01 \\
1.14 \\
\end{array}$ & $\begin{array}{l}0.38 \\
0.01 \\
-0.435 \\
\end{array}$ & $\begin{array}{l}0.69 \\
0 \\
0.93 \\
\end{array}$ & $\begin{array}{l}0.87 \\
0 \\
1.03 \\
\end{array}$ & $\begin{array}{l}0.57 \\
0 \\
2.14 \\
\end{array}$ & $\begin{array}{l}0.51 \\
0 \\
0.56 \\
\end{array}$ \\
\hline $\begin{array}{l}\mathrm{Fe}(\mathrm{III})_{\mathrm{fzn}} \\
(\mu \mathrm{M}) \\
2 \mathrm{~h}\end{array}$ & $\begin{array}{l}\mathrm{r}^{2} \\
\mathrm{p} \\
\mathrm{m}\end{array}$ & $\begin{array}{l}0.41 \\
0 \\
0.22\end{array}$ & & & & & & & & & $\begin{array}{l}0.22 \\
0.001 \\
0.83\end{array}$ & $\begin{array}{l}0.19 \\
0.09 \\
1.18\end{array}$ & $\begin{array}{l}0.91 \\
0 \\
1.24\end{array}$ & \\
\hline $\begin{array}{l}\text { Fe(tot })_{\text {fzn }} \\
(\mu \mathrm{M}) \\
2 \mathrm{~h}\end{array}$ & $\begin{array}{l}\mathrm{r}^{2} \\
\mathrm{p} \\
\mathrm{m}\end{array}$ & $\begin{array}{l}0.23 \\
0 \\
0.30 \\
\end{array}$ & $\begin{array}{l}0.25 \\
0 \\
2.17 \\
\end{array}$ & $\begin{array}{l}0.41 \\
0 \\
1.35\end{array}$ & $\begin{array}{l}0.16 \\
0 \\
0.36 \\
\end{array}$ & & & & & & & & & \\
\hline $\begin{array}{l}\text { ICP Fe } \\
(\mu \mathrm{M})\end{array}$ & $\begin{array}{l}\mathrm{r}^{2} \\
\mathrm{p} \\
\mathrm{m}\end{array}$ & $\begin{array}{l}0.35 \\
0 \\
8.34 \\
\end{array}$ & $\begin{array}{l}0.23 \\
0 \\
12.9 \\
\end{array}$ & $\begin{array}{l}0.53 \\
0 \\
14.7\end{array}$ & $\begin{array}{l}0.37 \\
0 \\
0.06 \\
\end{array}$ & $\begin{array}{l}0.18 \\
0 \\
0.02 \\
\end{array}$ & $\begin{array}{l}0.52 \\
0 \\
0.05 \\
\end{array}$ & & & & & & & \\
\hline $\begin{array}{l}\text { ICP Cu } \\
(\mu \mathrm{M})\end{array}$ & $\begin{array}{l}\mathrm{r}^{2} \\
\mathrm{p} \\
\mathrm{m}\end{array}$ & $\begin{array}{l}0.48 \\
0 \\
3.41\end{array}$ & $\begin{array}{l}0.14 \\
0.01 \\
1.94\end{array}$ & $\begin{array}{l}0.14 \\
0.01 \\
1.66\end{array}$ & $\begin{array}{l}0.52 \\
0 \\
0.21\end{array}$ & & & & & & & & & \\
\hline $\begin{array}{l}\text { ICP Mn } \\
(\mu \mathrm{M})\end{array}$ & $\begin{array}{l}\mathrm{r}^{2} \\
\mathrm{p} \\
\mathrm{m}\end{array}$ & $\begin{array}{l}0.18 \\
0 \\
0.18 \\
\end{array}$ & $\begin{array}{l}0.36 \\
0 \\
0.76 \\
\end{array}$ & $\begin{array}{l}0.30 \\
0 \\
0.43 \\
\end{array}$ & $\begin{array}{l}0.18 \\
0 \\
1.46 \\
\end{array}$ & $\begin{array}{l}0.34 \\
0 \\
0.63 \\
\end{array}$ & & & & & & & & \\
\hline
\end{tabular}


Table S3. Correlations between soluble trace metals and elements at $24 \mathrm{~h}$.

Coefficient of determination $\left(\mathrm{r}^{2}\right)$ are shown for the entire data set. Bold and red values indicate a $\mathrm{p}$-value $<0.05$. Some metals have fewer data points, thus the number of samples $p$ value thresholds vary depending on the metal or element.

\begin{tabular}{|c|c|c|c|c|c|c|c|c|c|c|c|}
\hline & $\mathrm{Cr}$ & Mn & Fe & $\mathrm{Ni}$ & $\mathrm{Cu}$ & Zn & Se & $\mathrm{Pb}$ & $\mathrm{Fe}(\mathrm{II})_{\mathrm{fzn}}$ & $\mathrm{Fe}(\text { tot })_{\mathrm{fzn}}$ & mass \\
\hline $\mathbf{V}$ & 0.07 & 0.06 & 0.10 & 0.03 & 0.41 & 0.02 & 0.20 & 0.09 & 0 & 0.03 & 0.32 \\
\hline $\mathrm{Cr}$ & & 0.55 & 0.61 & 0.21 & 0.26 & 0.08 & 0.04 & 0.05 & 0.05 & 0.13 & 0.17 \\
\hline Mn & & & 0.83 & 0.008 & 0.35 & 0.25 & 0.01 & 0.10 & 0.28 & 0.39 & 0.18 \\
\hline $\mathrm{Fe}$ & & & & 0.005 & 0.61 & 0.15 & 0.09 & 0.10 & 0.15 & 0.31 & 0.36 \\
\hline $\mathbf{N i}$ & & & & & 0.003 & 0.006 & 0.003 & 0.004 & 0.006 & 0.004 & 0.0 \\
\hline $\mathrm{Cu}$ & & & & & & 0.07 & 0.19 & 0.18 & 0.06 & 0.18 & 0.48 \\
\hline $\mathrm{Zn}$ & & & & & & & 0.01 & 0.15 & 0.11 & 0.12 & 0.07 \\
\hline Se & & & & & & & & 0.14 & 0.005 & 0.06 & 0.50 \\
\hline $\mathrm{Pb}$ & & & & & & & & & 0.21 & 0.18 & 0.16 \\
\hline $\mathrm{Fe}(\mathrm{II})_{\mathrm{fzn}}$ & & & & & & & & & & 0.71 & 0.03 \\
\hline $\mathrm{Fe}\left(\right.$ tot $_{\mathrm{fzn}}$ & & & & & & & & & & & 0.14 \\
\hline
\end{tabular}


a. Initial $\mathrm{OH}$ and wind directions by time of day
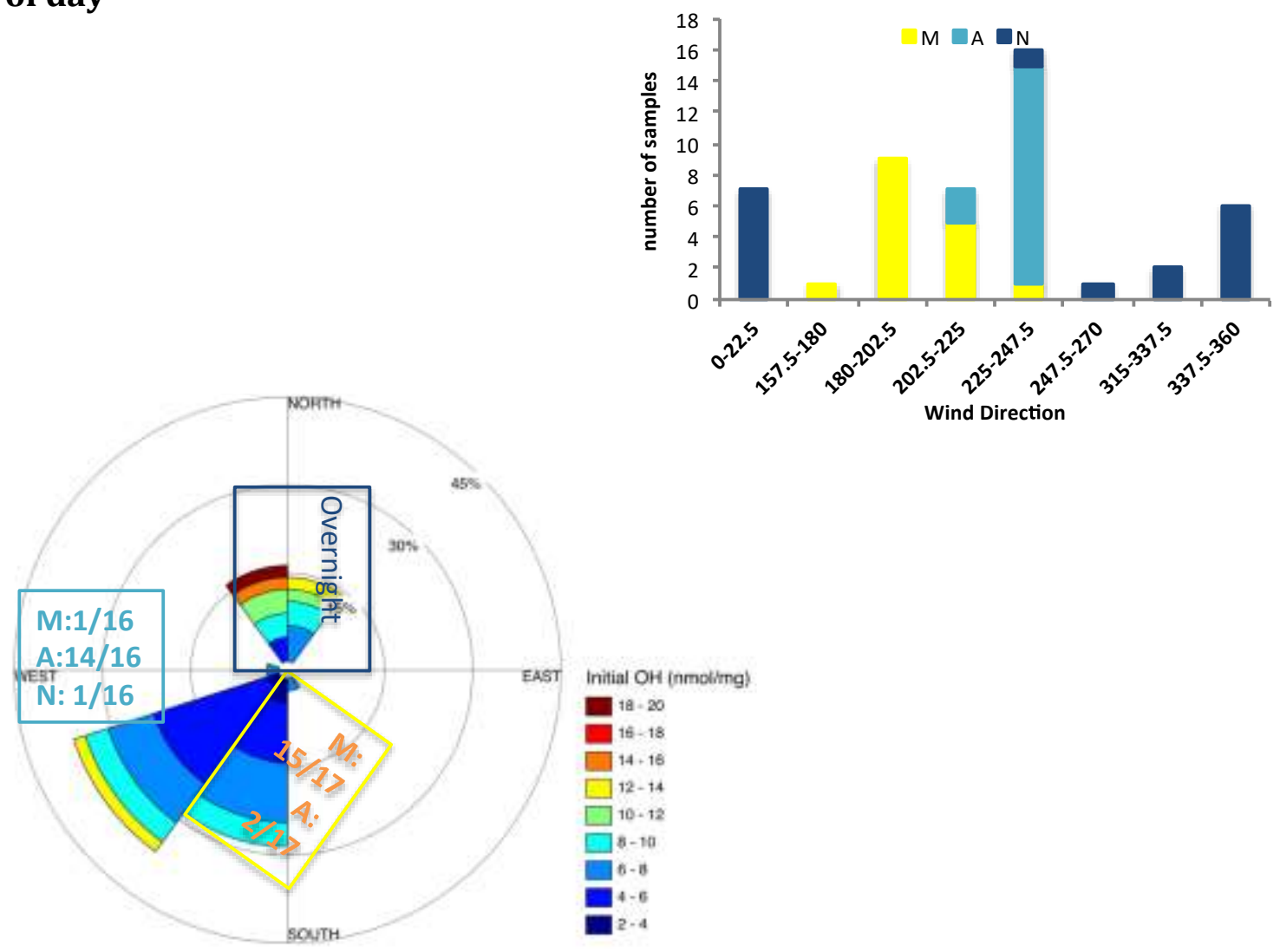

b. Fe(tot)fzn, 24 h

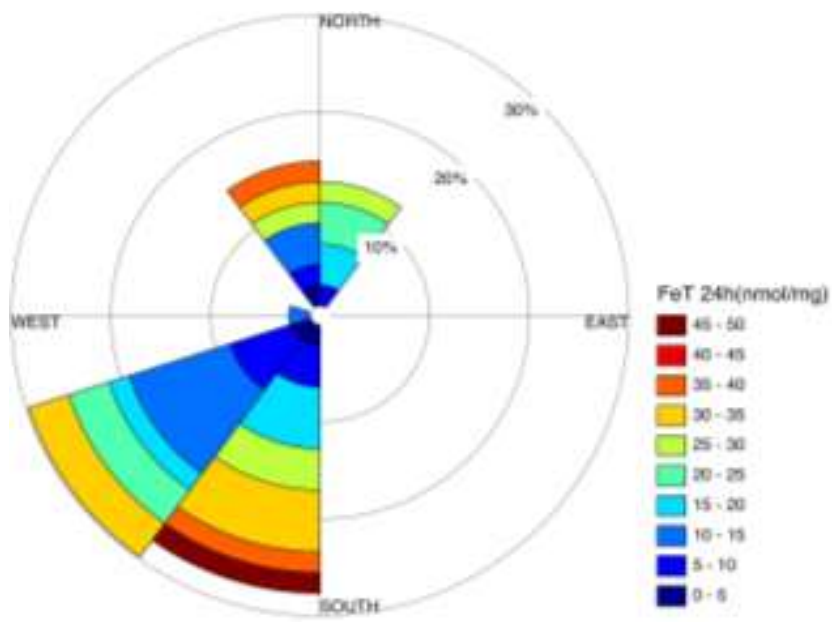


c. ICP Fe, 24 h

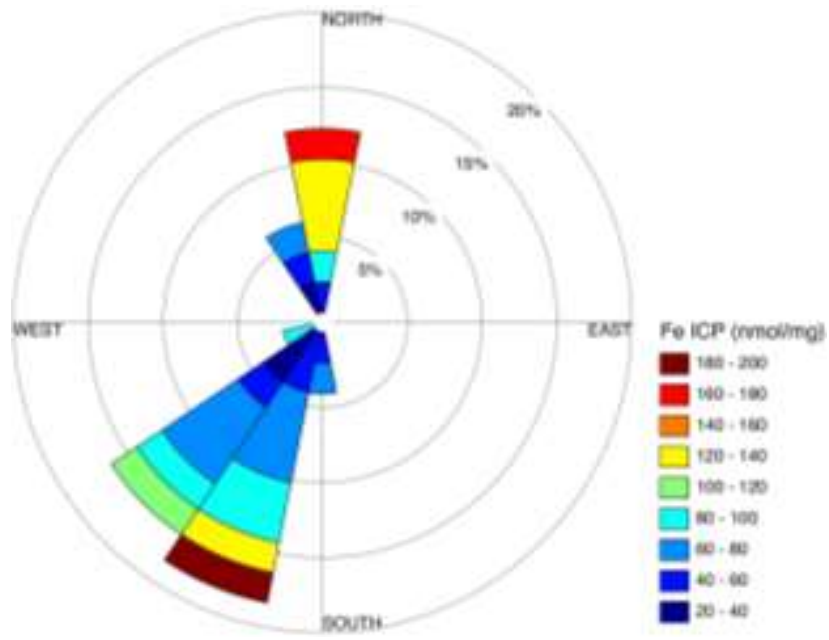

d. ICP Cu, 24 h

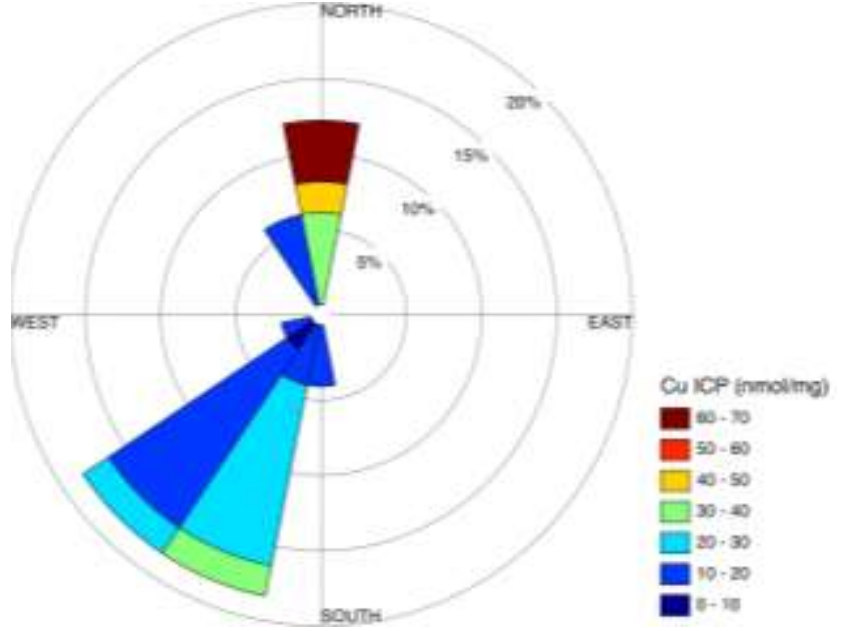

e. Anthraquinone

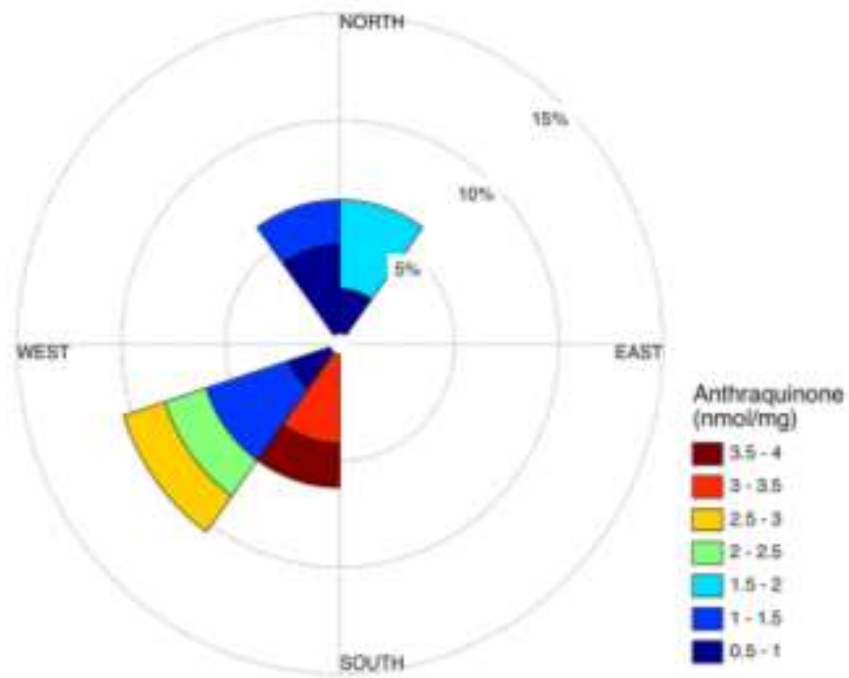




\section{f. Benz-7,12 anthraquinone}

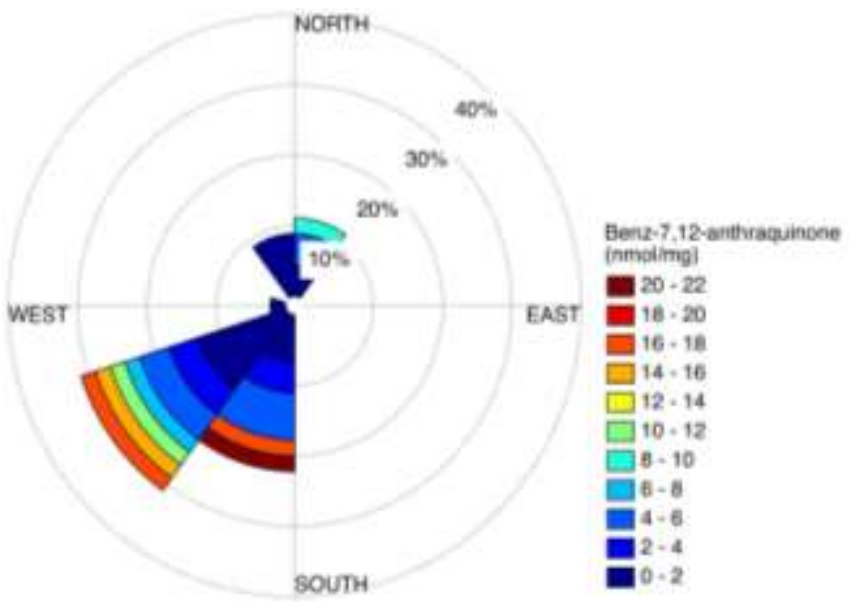

\section{g. Naphthacenequinone}

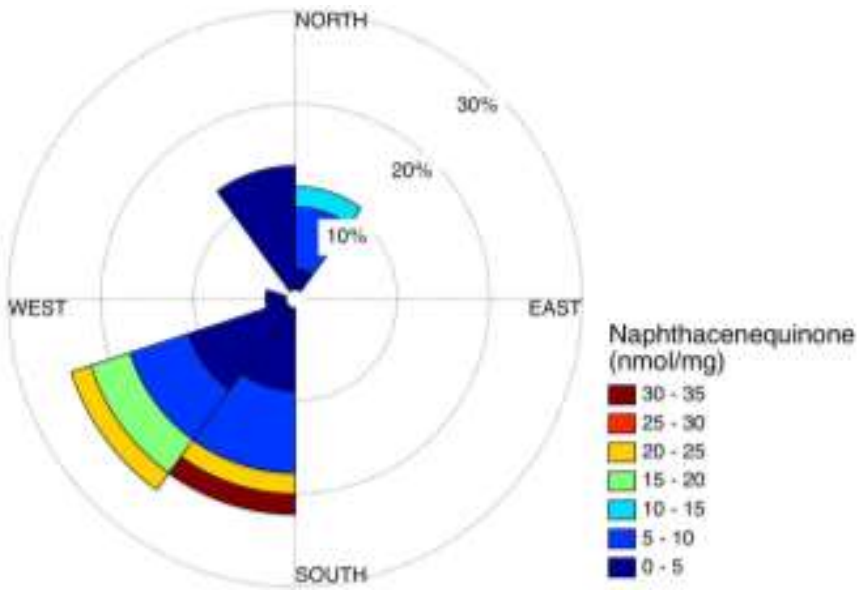

\section{h. 1,4-Naphthoquinone}

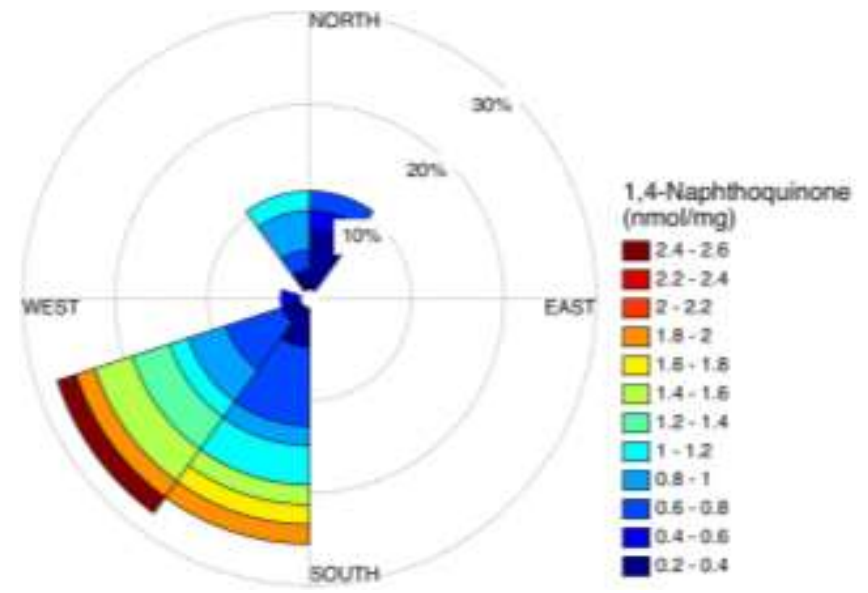

Figure S4. Wind roses showing $\mathrm{OH}$, quinone and soluble metal concentrations as a function of average wind direction. Percentage on the wind rose indicates the quantity of samples coming from each wind direction. Panel (a) also shows wind direction distribution by time of day. (a) Initial OH; (b) Fe(tot)fzn at 24 hours; (c) ICP $\mathrm{Fe}$; (d) ICP Cu; (e) anthraquinone; (f) benz-7,12-anthraquinone; (g) naphthacenequinone; and (h) 1,4 naphthaquinone. 


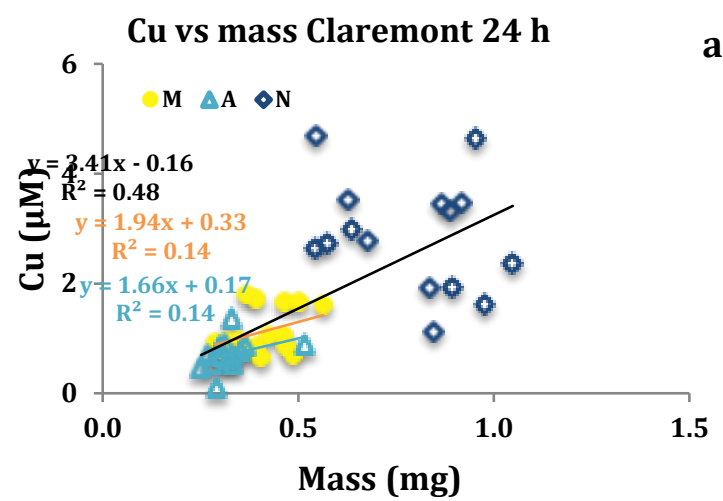

$\mathbf{a}$
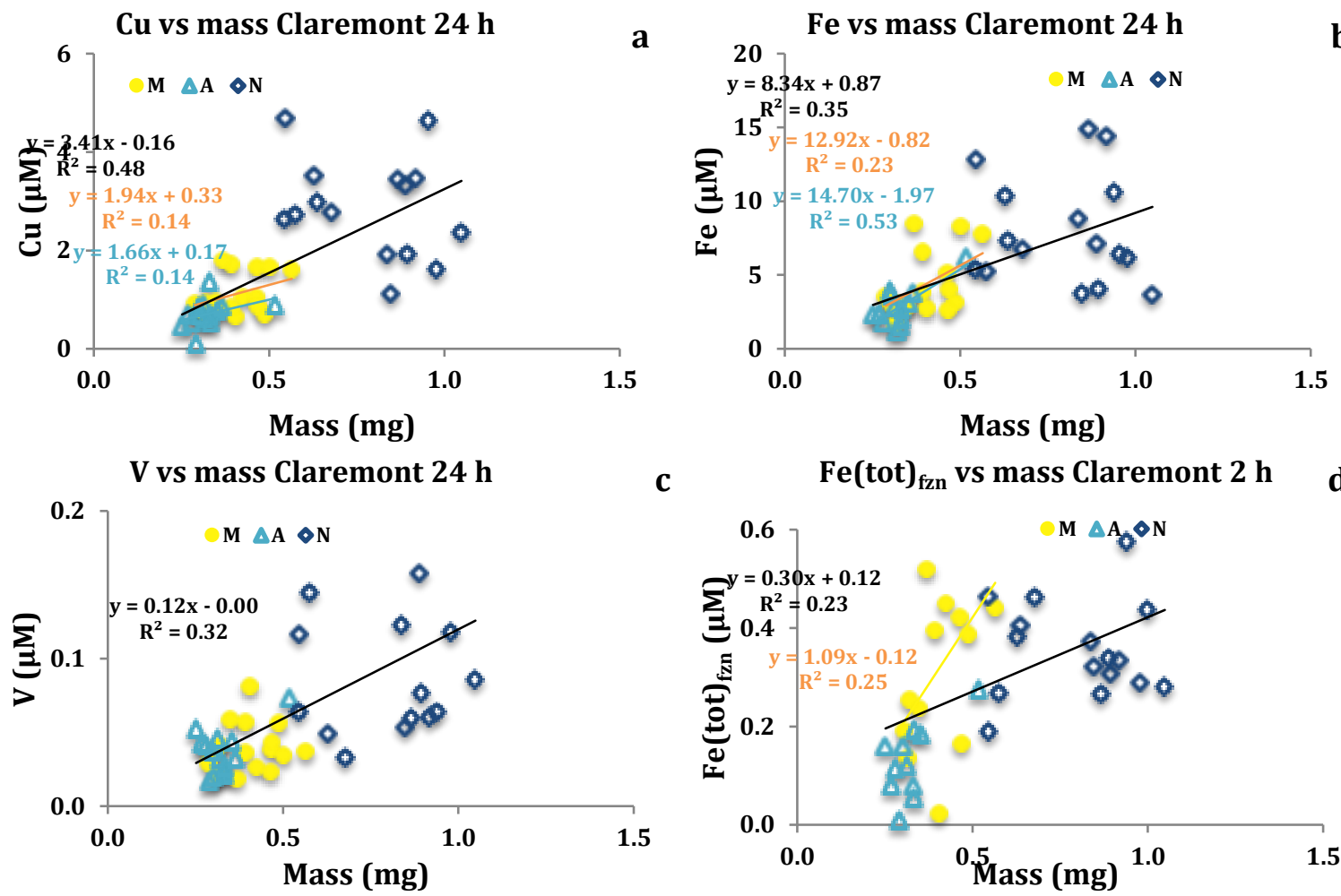

c
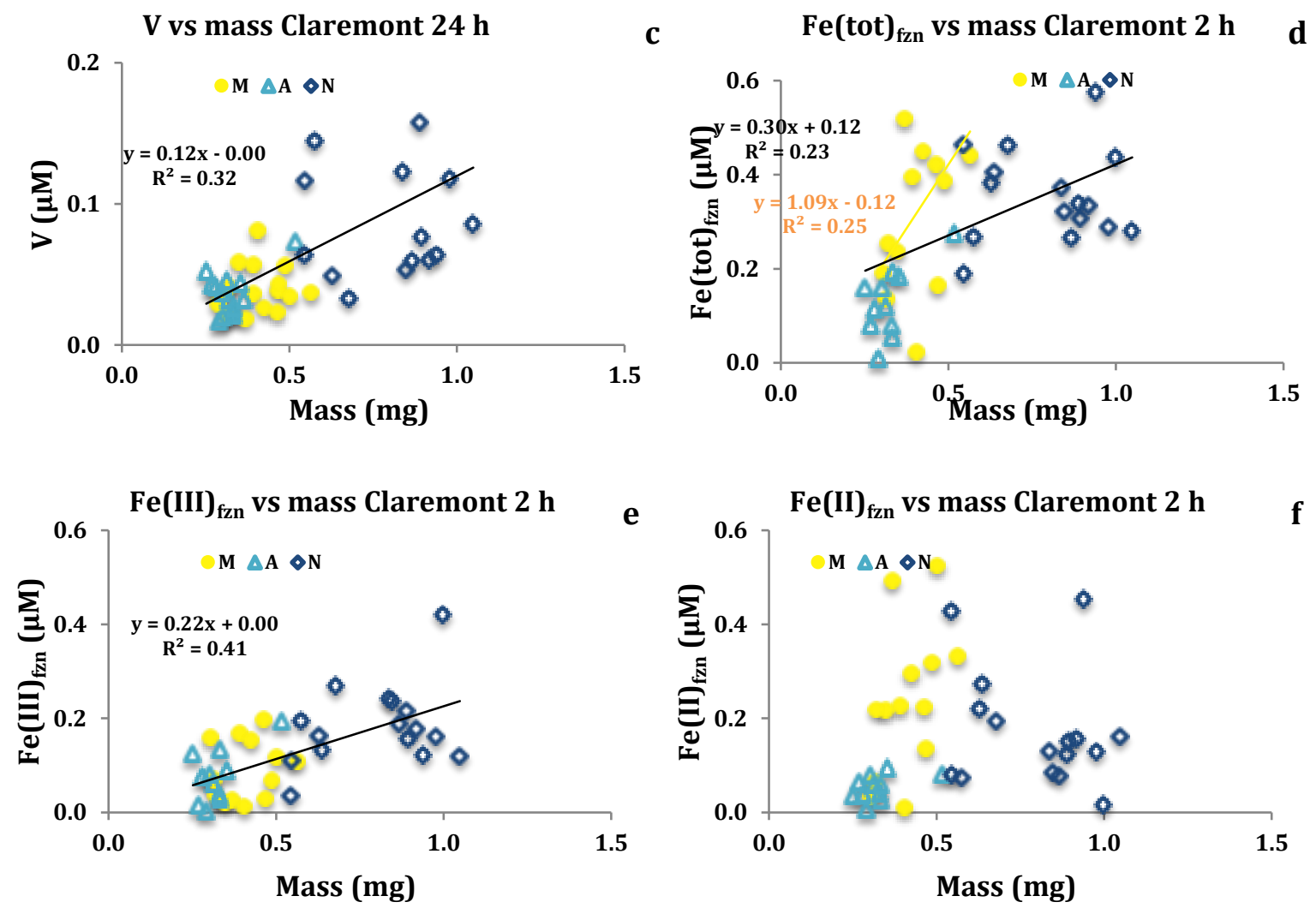

Figure S5. Relationships between $24 \mathrm{hr}$ soluble copper (a), iron (b) and vanadium (c) and aerosol mass, and ferrozine-measured total iron (Fe(tot) fzn (d), $\mathrm{Fe}(\mathrm{III})_{\mathrm{fzn}}$ (e), and Fe(II) fzn after 2 hours (f). Circles (M), triangle (A) and diamonds (N) represent morning, afternoon and nighttime samples, respectively. Black solid lines, equations and $\mathrm{r}^{2}$ indicate the slope of all data combined. Colored lines/text indicate statistics for corresponding subsets of the data (morning, afternoon or overnight). 